\title{
A locally commutative free group acting on the plane
}

\author{
by
}

Kenzi Satô (Tokyo)

\begin{abstract}
The purpose of this paper is to prove the existence of a free subgroup of the group of all affine transformations on the plane with determinant 1 such that the action of the subgroup is locally commutative.
\end{abstract}

Introduction. We say that a group $G$ of transformations is locally commutative if $\gamma \gamma^{\prime}=\gamma^{\prime} \gamma$ for any $\gamma, \gamma^{\prime} \in G$ such that $\gamma(\vec{z})=\vec{z}=\gamma^{\prime}(\vec{z})$ for some point $\vec{z}$. We will study the group $S A_{2}(\mathbb{R})$ of affine area-preserving and orientation-preserving transformations on the plane $\mathbb{R}^{2}$, that is, transformations of the form

$$
\left(\begin{array}{l}
x \\
y
\end{array}\right) \mapsto\left(\begin{array}{ll}
a & b \\
c & d
\end{array}\right)\left(\begin{array}{l}
x \\
y
\end{array}\right)+\left(\begin{array}{l}
p \\
q
\end{array}\right)
$$

where $a d-b c=1$. We will prove that there exists a free non-abelian locally commutative subgroup of $S A_{2}(\mathbb{R})$. More precisely:

THEOREM. For every transcendental real number $\theta$, the affine transformations

$$
u: \vec{z} \mapsto\left(\begin{array}{cc}
2 & \theta \\
0 & \frac{1}{2}
\end{array}\right) \vec{z}+\left(\begin{array}{c}
-1 \\
0
\end{array}\right) \quad \text { and } \quad v: \vec{z} \mapsto\left(\begin{array}{cc}
2 & 0 \\
\theta & \frac{1}{2}
\end{array}\right) \vec{z}+\left(\begin{array}{c}
0 \\
-\frac{1}{2}
\end{array}\right)
$$

are free generators of a group of transformations which is locally commutative.

By this Theorem (see the last section of our paper) the set of $n$-tuples $\left(\alpha_{0}, \ldots, \alpha_{n-1}\right)$ in $\left(S A_{2}(\mathbb{R})\right)^{n}$ which fail to be free generators of a free locally commutative subgroup is a subset of a countable union of proper algebraic subsets. It follows of course that this union is meager and of measure 0 . This has an additional consequence (for the proof see $\left[\mathrm{M}_{1}\right]$, for related general results see $\left[\mathrm{M}_{2}\right]$ and $\left.[\mathrm{K}]\right)$ :

2000 Mathematics Subject Classification: Primary 20E05, 28D05; Secondary 20G20.

Key words and phrases: free group, Hausdorff-Banach-Tarski Paradox. 
COROLlary. There exists a set $F \subseteq S A_{2}(\mathbb{R})$ which generates freely a locally commutative subgroup such that for every open non-empty subset $V$ of $S A_{2}(\mathbb{R}), F \cap V$ contains a perfect set (that is, non-empty, closed and dense in itself).

Remark. Of course $F$ has power continuum. However, by a well known theorem of Banach, $F$ or even any Borel set of free generators must be meager and of measure 0 .

These results are motivated by a long line of research starting with Klein, Fricke, and Hausdorff and more specifically von Neumann [V] (see also [W, Th. 7.3], [MW, $\left.\mathrm{M}_{3}, \mathrm{~L}, \mathrm{~S}_{0}\right]$ ). He proved that every bounded subset $S$ of $\mathbb{R}^{2}$ with non-empty interior is $S A_{2}(\mathbb{R})$-paradoxical, that is, for some positive integers $N$ and $N^{\prime}$ there are pairwise disjoint subsets $S_{0}, \ldots, S_{N+N^{\prime}-1}$ of $S$ and $\gamma_{0}, \ldots, \gamma_{N+N^{\prime}-1} \in S A_{2}(\mathbb{R})$ such that

$$
S=\gamma_{0}\left(S_{0}\right) \cup \ldots \cup \gamma_{N-1}\left(S_{N-1}\right)=\gamma_{N}\left(S_{N}\right) \cup \ldots \cup \gamma_{N+N^{\prime}-1}\left(S_{N+N^{\prime}-1}\right) .
$$

Von Neumann's proof requires only the existence of a free non-abelian subgroup of $S A_{2}(\mathbb{R})$. But other results require local commutativity. For example the above corollary implies that there exists a set $E \subseteq \mathbb{R}^{2}$ such that for every countable set $D \subseteq \mathbb{R}^{2}$ there exists a $\gamma \in S A_{2}(\mathbb{R})$ such that

$$
\gamma(E)=E \triangle D,
$$

where $\triangle$ denotes the symmetric difference of sets (for the proof see $\left[\mathrm{M}_{0}\right]$ ). Also, it implies a very general theorem about the existence of partitions of $\mathbb{R}^{2}$ into disjoint sets $S_{j}(j \in I)$ satisfying certain systems of equations of the form

$$
\alpha\left(\bigcup_{j \in I_{\alpha}} S_{j}\right)=\bigcup_{j^{\prime} \in I_{\alpha}^{\prime}} S_{j^{\prime}},
$$

where $\alpha$ runs over our set $F$ of free generators of a locally commutative group, and $\emptyset \neq I_{\alpha}, I_{\alpha}^{\prime} \subsetneq I$. The only requirement is that the consequences of this system obtained by complementation of both sides of an equation and by transitivity do not yield an equation of the form $\gamma(S)=\mathbb{R}^{2} \backslash S$ (for the proof see [W, Corollary 4.12]). A special case is a partition of $\mathbb{R}^{2}$ into four sets $S_{0}, S_{1}, S_{2}$, and $S_{3}$ satisfying

$$
u\left(S_{1}\right)=S_{1} \cup S_{2} \cup S_{3}, \quad v\left(S_{3}\right)=S_{0} \cup S_{1} \cup S_{3},
$$

where $u$ and $v$ are as in our Theorem. Thus, each of the sets $S_{0} \cup S_{1}$ and $S_{2} \cup S_{3}$ yields the whole plane $\mathbb{R}^{2}$ by decomposition into two pieces and transformation of one of them. For more applications similar to the above, see $[\mathrm{W}]$.

Finally let us add that, in connection with the above mentioned theorem of von Neumann, M. Laczkovich [L] proved that $] 0,1] \times] 0,1]$ is $S L_{2}(\mathbb{R})$ paradoxical, where $S L_{2}(\mathbb{R})$ is the subgroup of $S A_{2}(\mathbb{R})$ consisting of lin- 
ear transformations. And the author has shown in $\left[\mathrm{S}_{0}\right]$ that a subgroup of $S A_{2}(\mathbb{R})$ is freely generated by the transformations

$$
\begin{aligned}
\zeta\left(\begin{array}{l}
x \\
y
\end{array}\right) & =\left(\begin{array}{ll}
7 & 3 \\
9 & 4
\end{array}\right)\left(\begin{array}{l}
x \\
y
\end{array}\right)+\left(\begin{array}{c}
1 \\
-1
\end{array}\right) \\
\eta\left(\begin{array}{l}
x \\
y
\end{array}\right) & =\left(\begin{array}{cc}
94 & 39 \\
147 & 61
\end{array}\right)\left(\begin{array}{l}
x \\
y
\end{array}\right)+\left(\begin{array}{l}
3 \\
2
\end{array}\right)
\end{aligned}
$$

and this subgroup acts without fixed points on $\mathbb{Z}^{2}$ (that is, $\gamma(\vec{z}) \neq \vec{z}$ for all $\vec{z} \in \mathbb{Z}^{2}$ and $\gamma$ different from the identity).

The author is grateful to Jan Mycielski who read the earlier versions of this paper, gave many helpful remarks and suggested the above Corollary.

Proof of Theorem. The first part of the proof, beginning with Lemma 0 and up to Proposition 0 , will be parallel to an argument in $\left[\mathrm{S}_{1}\right]$; however, the calculations are rather different. The remainder of the proof takes advantage of Lemmas 3-5, Proposition 0 and Theorem 3 of $\left[\mathrm{S}_{1}\right]$. Then we need a new Lemma 2 which is used in the final argument, the proof of local commutativity.

Let $u$ and $v$ be elements of $S A_{2}(\mathbb{R})$, i.e.,

$$
u: \vec{z} \mapsto A \vec{z}+\vec{p}, \quad v: \vec{z} \mapsto B \vec{z}+\vec{q},
$$

where $A$ and $B$ are $2 \times 2$ matrices with determinant 1 and $\vec{p}$ and $\vec{q}$ are vectors in $\mathbb{R}^{2}$.

Lemma 0. Let

$$
w=u^{k_{0}} v^{l_{0}} u^{k_{1}} v^{l_{1}} \ldots u^{k_{m-1}} v^{l_{m-1}},
$$

where $m$ is a positive integer and $k_{0}, l_{0}, k_{1}, l_{1}, \ldots, k_{m-1}, l_{m-1}$ are non-zero integers. If the matrices

$$
\text { id }-A, \quad \text { id }-B, \quad \text { id }-A^{k_{0}} B^{l_{0}} A^{k_{1}} B^{l_{1}} \ldots A^{k_{m-1}} B^{l_{m-1}}
$$

are invertible, then the vector

$$
\begin{aligned}
& \left(\mathrm{id}-A^{k_{0}} B^{l_{0}} A^{k_{1}} B^{l_{1}} \ldots A^{k_{m-1}} B^{l_{m-1}}\right)^{-1} \cdot\left(\mathrm{id}-A^{k_{0}}+A^{k_{0}} B^{l_{0}}-A^{k_{0}} B^{l_{0}} A^{k_{1}}+\ldots\right. \\
& \left.+A^{k_{0}} B^{l_{0}} \ldots B^{l_{m-2}}-A^{k_{0}} B^{l_{0}} \ldots B^{l_{m-2}} A^{k_{m-1}}\right) \cdot\left((\mathrm{id}-A)^{-1} \vec{p}-(\mathrm{id}-B)^{-1} \vec{q}\right) \\
& +(\mathrm{id}-B)^{-1} \vec{q}
\end{aligned}
$$

is the unique fixed point of the transformation $w$.

Proof. We have the equalities

$$
u^{k}(\vec{z})=A^{k} \vec{z}+\frac{\mathrm{id}-A^{k}}{\mathrm{id}-A} \cdot \vec{p}, \quad v^{l}(\vec{z})=B^{l} \vec{z}+\frac{\mathrm{id}-B^{l}}{\mathrm{id}-B} \cdot \vec{q} .
$$


K. Satô

\section{Hence}

$$
\begin{aligned}
w(\vec{z}) & A^{k_{0}}\left(B^{l_{0}}\left(\ldots\left(A^{k_{m-1}}\left(B^{l_{m-1}} \vec{z}+\frac{\mathrm{id}-B^{l_{m-1}}}{\mathrm{id}-B} \cdot \vec{q}\right)+\frac{\mathrm{id}-A^{k_{m-1}}}{\mathrm{id}-A} \cdot \vec{p}\right) \ldots\right)\right. \\
& \left.+\frac{\mathrm{id}-B^{l_{0}}}{\mathrm{id}-B} \cdot \vec{q}\right)+\frac{\mathrm{id}-A^{k_{0}}}{\mathrm{id}-A} \vec{p} \\
= & A^{k_{0}} B^{l_{0}} \ldots B^{l_{m-1}} \vec{z}+\left(\left(\mathrm{id}-A^{k_{0}}\right)+A^{k_{0}} B^{l_{0}}\left(\mathrm{id}-A^{k_{1}}\right)+\ldots\right. \\
& \left.+A^{k_{0}} B^{l_{0}} \ldots B^{l_{m-2}}\left(\mathrm{id}-A^{k_{m-1}}\right)\right) \cdot(\mathrm{id}-A)^{-1} \cdot \vec{p} \\
& +\left(A^{k_{0}}\left(\mathrm{id}-B^{l_{0}}\right)+A^{k_{0}} B^{l_{0}} A^{k_{1}}\left(\mathrm{id}-B^{l_{1}}\right)+\ldots\right. \\
& \left.+A^{k_{0}} B^{l_{0}} \ldots A^{k_{m-1}}\left(\mathrm{id}-B^{l_{m-1}}\right)\right) \cdot(\mathrm{id}-B)^{-1} \cdot \vec{q} \\
= & A^{k_{0}} B^{l_{0}} \ldots B^{l_{m-1}} \vec{z}+\left(\mathrm{id}-A^{k_{0}}+A^{k_{0}} B^{l_{0}}-A^{k_{0}} B^{l_{0}} A^{k_{1}}+\ldots\right. \\
& \left.+A^{k_{0}} B^{l_{0}} \ldots B^{l_{m-2}}-A^{k_{0}} B^{l_{0}} \ldots A^{k_{m-1}}\right) \cdot\left((\mathrm{id}-A)^{-1} \vec{p}-(\mathrm{id}-B)^{-1} \vec{q}\right) \\
& +\left(\mathrm{id}-A^{k_{0}} B^{l_{0}} \ldots B^{l_{m-1}}\right) \cdot(\mathrm{id}-B)^{-1} \cdot \vec{q},
\end{aligned}
$$

which implies immediately the conclusion of the lemma.

From now on, let $A, \vec{p}, B, \vec{q}$ be as in the Theorem, i.e.,

$$
\begin{array}{ll}
A=\left(\begin{array}{cc}
2 & \frac{3}{2} \tau \\
0 & \frac{1}{2}
\end{array}\right), & \vec{p}=\left(\begin{array}{c}
-1 \\
0
\end{array}\right), \\
B=\left(\begin{array}{cc}
2 & 0 \\
\frac{3}{2} \tau & \frac{1}{2}
\end{array}\right), & \vec{q}=\left(\begin{array}{c}
0 \\
-\frac{1}{2}
\end{array}\right),
\end{array}
$$

where $\tau=\frac{2}{3} \theta$.

Lemma 1. Let $w$ be a non-empty reduced word in $u$ and $v$. Then the diagonal elements of the matrix part in $w$ are polynomials in $\mathbb{Q}\left[\tau^{2}\right]$ and other elements are polynomials of the form $\tau \cdot f$, where $f \in \mathbb{Q}\left[\tau^{2}\right]$. Moreover:

- if $w=u^{k_{0}} v^{l_{0}} \ldots v^{l_{m-1}}(m \geq 1)$ then the highest terms of the matrix part of $w$ are

$A^{k_{0}} B^{l_{0}} \ldots B^{l_{m-1}}$

$$
=\left(\begin{array}{ll}
\ldots+t\left(k_{0}\right) t\left(l_{0}\right) \ldots t\left(l_{m-1}\right) \tau^{2 m} & \ldots+t\left(k_{0}\right) t\left(l_{0}\right) \ldots t\left(k_{m-1}\right) 2^{-l_{m-1}} \tau^{2 m-1} \\
\ldots+2^{-k_{0}} t\left(l_{0}\right) \ldots t\left(l_{m-1}\right) \tau^{2 m-1} & \ldots+2^{-k_{0}} t\left(l_{0}\right) \ldots t\left(k_{m-1}\right) 2^{-l_{m-1}} \tau^{2 m-2}
\end{array}\right),
$$

- if $w=u^{k_{0}} v^{l_{0}} \ldots u^{k_{m}}(m \geq 0)$ then the highest terms of the matrix part of $w$ are 


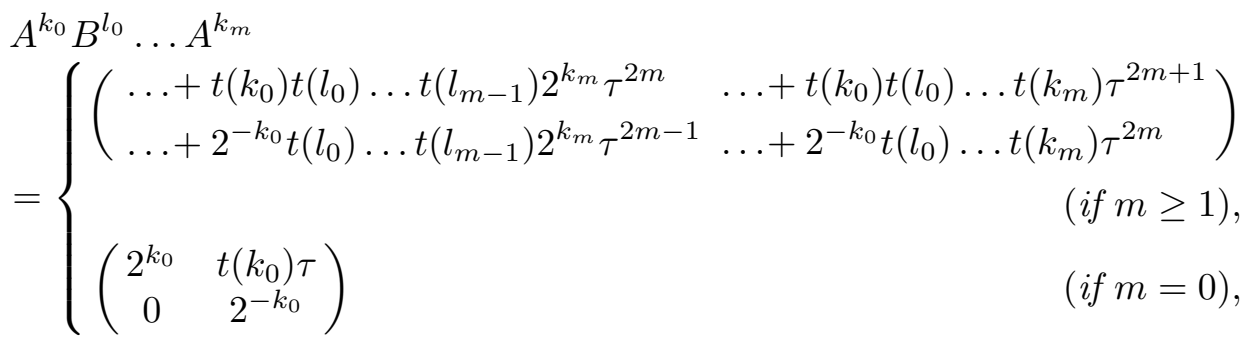

- if $w=v^{l_{0}} u^{k_{1}} \ldots u^{k_{m}}(m \geq 1)$ then the highest terms of the matrix part of $w$ are

$B^{l_{0}} A^{k_{1}} \ldots A^{k_{m}}$

$=\left(\begin{array}{ll}\ldots+2^{l_{0}} t\left(k_{1}\right) \ldots t\left(l_{m-1}\right) 2^{k_{m}} \tau^{2 m-2} & \ldots+2^{l_{0}} t\left(k_{1}\right) \ldots t\left(k_{m}\right) \tau^{2 m-1} \\ \ldots+t\left(l_{0}\right) t\left(k_{1}\right) \ldots t\left(l_{m-1}\right) 2^{k_{m}} \tau^{2 m-1} & \ldots+t\left(l_{0}\right) t\left(k_{1}\right) \ldots t\left(k_{m}\right) \tau^{2 m}\end{array}\right)$,

- if $w=v^{l_{0}} u^{k_{1}} \ldots v^{l_{m}}(m \geq 0)$ then the highest terms of the matrix part of $w$ are $B^{l_{0}} A^{k_{1}} \ldots B^{l_{m}}$

$=\left\{\begin{array}{lr}\left(\begin{array}{lr}\ldots+2^{l_{0}} t\left(k_{1}\right) \ldots t\left(l_{m}\right) \tau^{2 m} & \ldots+2^{l_{0}} t\left(k_{1}\right) \ldots t\left(k_{m}\right) 2^{-l_{m}} \tau^{2 m-1} \\ \ldots+t\left(l_{0}\right) t\left(k_{1}\right) \ldots t\left(l_{m}\right) \tau^{2 m+1} & \ldots+t\left(l_{0}\right) t\left(k_{1}\right) \ldots t\left(k_{m}\right) 2^{-l_{m}} \tau^{2 m}\end{array}\right) \\ \left(\begin{array}{cc}2^{l_{0}} & 0 \\ t\left(l_{0}\right) \tau & 2^{-l_{0}}\end{array}\right) & (\text { if } m \geq 1) \\ & (\text { if } m=0)\end{array}\right.$ where $t(k)=2^{k}-2^{-k}$.

Proof. This lemma is shown inductively by simple calculations (the proof is similar to the proof of Lemma 1 in $\left[\mathrm{S}_{1}\right]$, but the calculations are simpler).

Lemma 1 implies immediately the following facts:

$$
\begin{aligned}
\operatorname{det}\left(\mathrm{id}-A^{k_{0}}\right) & =2-\operatorname{tr}\left(A^{k_{0}}\right)=-\left(2^{k_{0}}-1\right)\left(1-2^{-k_{0}}\right) \neq 0, \\
\operatorname{det}\left(\mathrm{id}-B^{l_{0}}\right) & =2-\operatorname{tr}\left(B^{l_{0}}\right)=-\left(2^{l_{0}}-1\right)\left(1-2^{-l_{0}}\right) \neq 0, \\
\operatorname{det}\left(\mathrm{id}-A^{k_{0}} B^{l_{0}} \ldots B^{l_{m-1}}\right) & =2-\operatorname{tr}\left(A^{k_{0}} B^{l_{0}} \ldots B^{l_{m-1}}\right) \\
& =\ldots-t\left(k_{0}\right) \ldots t\left(l_{m-1}\right) \tau^{2 m} \neq 0 .
\end{aligned}
$$

In other words, each word $w=u^{k_{0}} v^{l_{0}} \ldots v^{l_{m-1}}$ satisfies the assumption of Lemma 0 . Hence the fixed point of $w$ is unique. The fixed point of a power of $u$ or of $v$ is also unique, so the fixed point of each non-empty reduced word is unique. So the first part of our Theorem follows: The group $F_{2}$ generated by $u$ and $v$ is a free group of rank 2 . Therefore we can define an equivalence relation on all non-empty reduced words in $u$ and $v$,

$$
w \sim w^{\prime} \underset{\text { def }}{\Longleftrightarrow} w \text { and } w^{\prime} \text { have a common fixed point in } \mathbb{R}^{2},
$$

and the following Proposition 0 of $\left[\mathrm{S}_{1}\right]$ is true: 
Proposition 0. For non-zero integers $k$ and $l$, we have

$$
w^{k} \sim w^{\prime l} \Leftrightarrow w \sim w^{\prime} \Leftrightarrow \bar{w} w \bar{w}^{-1} \sim \bar{w} w^{\prime} \bar{w}^{-1} .
$$

Furthermore, if $w^{-1} \neq w^{\prime}$, we have

$$
w \sim w^{\prime} w \Leftrightarrow w \sim w^{\prime} \Leftrightarrow w \sim w w^{\prime} .
$$

Let us divide cyclically reduced words of $F_{2}$ into six different types according to their first and last letters up to inversions:

$$
\begin{aligned}
& u \ldots u, \quad u^{-1} \ldots v^{-1}, \quad u^{-1} \ldots v, \\
& v \ldots v, \quad u \ldots v^{-1}, \quad u \ldots v .
\end{aligned}
$$

Using Lemmas $3-5$ and Theorem 3 of $\left[\mathrm{S}_{1}\right]$, we see that in order to prove that the action of $F_{2}$ on $\mathbb{R}^{2}$ is locally commutative it is enough to show that words of distinct types $(*)$ have no common fixed points. From now on, our proof diverges from that of $\left[\mathrm{S}_{1}\right]$. Consider the following four types:

$$
\begin{array}{cc}
u^{-1} \ldots v^{-1}, & u^{-1} \ldots v, \\
u \ldots v^{-1}, & u \ldots v .
\end{array}
$$

LEMma 2. If any two reduced words of distinct types of $(\diamond)$ have no common fixed points in $\mathbb{R}^{2}$, then the same is true for reduced words of distinct types of $(*)$.

Proof. Let $\nsim$ denote the negation of $\sim$. We will argue by induction relative to the length of reduced words. For $w=u \ldots u$ and $w^{\prime}=v \ldots v$, from the assumption of this lemma, we have $w^{-1} w^{\prime} \not w w^{\prime}$. Thus $w \not w^{\prime}$ (since if $w \sim w^{\prime}$, then from Proposition 0 we have $w^{-1} w^{\prime} \sim w^{\prime} \sim w w^{\prime}$, a contradiction). For $w=u \ldots u$ and $w^{\prime \prime}=u^{\varepsilon} \ldots v^{\delta}$ with $\varepsilon, \delta \in\{-1,1\}$, let $\widetilde{w}=w^{-\varepsilon} w^{\prime \prime}$. If $w^{\varepsilon} u^{\varepsilon} \subseteq w^{\prime \prime}$ (where $w_{0} \subseteq w_{1}$ means $w_{0}$ is an initial segment of $w_{1}$, i.e., we can represent $w_{1}$ in the form $w_{0} \bar{w}$ without cancellation), then we can reduce $w \not w^{\prime \prime}$ to $w \nsim \widetilde{w}$ with shorter length. So, applying the inductive assumption, we can assume that $w^{\varepsilon} u^{\varepsilon} \nsubseteq w^{\prime \prime}$. If $w^{\varepsilon} \subseteq w^{\prime \prime}$, i.e., $w^{\varepsilon} v^{-1} \subseteq w^{\prime \prime}$ or $w^{\varepsilon} v \subseteq w^{\prime \prime}$, then $w^{-\varepsilon} \widetilde{w} \nsim w^{\prime \prime}$. If $w^{\varepsilon} \supseteq w^{\prime \prime} u^{\varepsilon}$, then we can reduce the relation $w \nsim w^{\prime \prime}$ to $\widetilde{w}^{-\varepsilon} \nsim w^{\prime \prime}$ with shorter length. So we can assume $w^{\varepsilon} \nsupseteq w^{\prime \prime} u^{\varepsilon}$. If $w^{\varepsilon} \supseteq w^{\prime \prime}$, i.e., $w^{\varepsilon} \supseteq w^{\prime \prime} u^{-\varepsilon}$ or $w^{\varepsilon} \supseteq w^{\prime \prime} v^{\delta}$, then $\widetilde{w} w^{\prime \prime} \chi w^{\prime \prime}$. Otherwise, i.e., if neither $w^{\varepsilon} \subseteq w^{\prime \prime}$ nor $w^{\varepsilon} \supseteq w^{\prime \prime}$, we have $\widetilde{w} \not w^{\prime \prime}$. For $w^{\prime}=v \ldots v$ and $w^{\prime \prime}=u^{\varepsilon} \ldots v^{\delta}$, the proof is similar.

Now we will prove the second part of the Theorem, which states that: The group $F_{2}$ acts on $\mathbb{R}^{2}$ in a locally commutative way. By Lemma 2 this follows immediately from the following:

Lemma 3. Words of distinct types $(\diamond)$ have no common fixed points.

Proof. For $w=u^{k_{0}} v^{l_{0}} \ldots v^{l_{m-1}}$, we define 


$$
\begin{aligned}
\vec{r}(w) & =\left(2-\operatorname{tr}\left(A^{k_{0}} B^{l_{0}} \ldots B^{l_{m-1}}\right)\right) \cdot\left((\text { the fixed point of } w)-(\mathrm{id}-B)^{-1} \cdot \vec{q}\right) \\
= & \left(\mathrm{id}-\left(A^{k_{0}} B^{l_{0}} \ldots B^{l_{m-1}}\right)^{-1}\right) \cdot\left(\mathrm{id}-A^{k_{0}}+A^{k_{0}} B^{l_{0}}-A^{k_{0}} B^{l_{0}} A^{k_{1}}+\ldots\right. \\
& \left.+A^{k_{0}} B^{l_{0}} \ldots B^{l_{m-2}}-A^{k_{0}} B^{l_{0}} \ldots A^{k_{m-1}}\right) \cdot\left((\mathrm{id}-A)^{-1} \vec{p}-(\mathrm{id}-B)^{-1} \vec{q}\right) \\
= & \left(\mathrm{id}-A^{k_{0}}+A^{k_{0}} B^{l_{0}}-A^{k_{0}} B^{l_{0}} A^{k_{1}}+\ldots\right. \\
& +A^{k_{0}} B^{l_{0}} \ldots B^{l_{m-2}}-A^{k_{0}} B^{l_{0}} \ldots A^{k_{m-1}} \\
& -B^{-l_{m-1}} A^{-k_{m-1}} \ldots A^{-k_{0}}+B^{-l_{m-1}} A^{-k_{m-1}} \ldots B^{-l_{0}}-\ldots \\
& \left.-B^{-l_{m-1}} A^{-k_{m-1}}+B^{-l_{m-1}}\right) \cdot\left((\mathrm{id}-A)^{-1} \vec{p}-(\mathrm{id}-B)^{-1} \vec{q}\right)
\end{aligned}
$$

where the second equality follows from Lemma 0 . Since

$$
2-\operatorname{tr}\left(A^{k_{0}} B^{l_{0}} \ldots B^{l_{m-1}}\right) \in \mathbb{Q}\left[\tau^{2}\right],
$$

it is enough to show that for two reduced words $w$ and $w^{\prime}$ of distinct types $(\diamond)$, the vectors $\vec{r}(w)$ and $\vec{r}\left(w^{\prime}\right)$ are linearly independent over $\mathbb{Q}\left(\tau^{2}\right)$. For $w=u^{k_{0}} v^{l_{0}} \ldots v^{l_{m-1}}$, we have

$$
\begin{aligned}
& \vec{r}(w)=\left(\ldots+A^{k_{0}} B^{l_{0}} \ldots B^{l_{m-2}}-A^{k_{0}} B^{l_{0}} \ldots A^{k_{m-1}}\right. \\
& \left.-B^{-l_{m-1}} A^{-k_{m-1}} \ldots A^{-k_{0}}+B^{-l_{m-1}} A^{-k_{m-1}} \ldots B^{-l_{0}}-\ldots\right) \\
& \cdot\left((\mathrm{id}-A)^{-1} \vec{p}-(\mathrm{id}-B)^{-1} \vec{q}\right)
\end{aligned}
$$

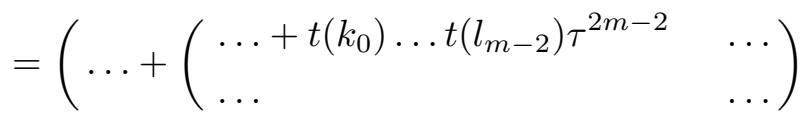

$$
\begin{aligned}
& -\left(\begin{array}{ll}
\ldots+t\left(k_{0}\right) \ldots t\left(l_{m-2}\right) 2^{k_{m-1}} \tau^{2 m-2} & \ldots+t\left(k_{0}\right) \ldots t\left(k_{m-1}\right) \tau^{2 m-1} \\
\ldots & \ldots
\end{array}\right) \\
& -\left(\begin{array}{cr}
\ldots+2^{-l_{m-1}} t\left(-k_{m-1}\right) \ldots & \ldots+2^{-l_{m-1}} t\left(-k_{m-1}\right) \ldots \\
\cdot t\left(-l_{0}\right) 2^{-k_{0}} \tau^{2 m-2} & \cdot t\left(-k_{0}\right) \tau^{2 m-1} \\
\ldots+t\left(-l_{m-1}\right) \ldots t\left(-l_{0}\right) 2^{-k_{0}} \tau^{2 m-1} & \ldots+t\left(-l_{m-1}\right) \ldots t\left(-k_{0}\right) \tau^{2 m}
\end{array}\right) \\
& \left.+\left(\begin{array}{ll}
\ldots+2^{-l_{m-1}} t\left(-k_{m-1}\right) \ldots t\left(-l_{0}\right) \tau^{2 m-2} & \ldots \\
\ldots+t\left(-l_{m-1}\right) \ldots t\left(-l_{0}\right) \tau^{2 m-1} & \ldots
\end{array}\right)-\ldots\right) \cdot\left(\begin{array}{l}
1 \\
1
\end{array}\right) \\
& =\left\{\begin{array}{r}
\cdots+t\left(l_{0}\right) \ldots t\left(l_{m-2}\right) \tau^{2 m-2} \\
\cdot\left(\begin{array}{r}
t\left(k_{0}\right)\left(1-2^{k_{m-1}}\right)+\left(-2^{-k_{0}}+1\right) t\left(k_{m-1}\right) 2^{-l_{m-1}} \\
+t\left(k_{0}\right) t\left(k_{m-1}\right)\left(-1+2^{-l_{m-1}}\right) \tau \\
\left(2^{-k_{0}}-1\right) t\left(k_{m-1}\right) t\left(l_{m-1}\right) \tau-t\left(k_{0}\right) t\left(k_{m-1}\right) t\left(l_{m-1}\right) \tau^{2}
\end{array}\right)
\end{array}\right. \\
& \ldots+\left(\begin{array}{c}
\left(1-2^{k_{0}}\right)+\left(-2^{-k_{0}}+1\right) 2^{-l_{0}}+t\left(k_{0}\right)\left(-1+2^{-l_{0}}\right) \tau \\
\left(2^{-k_{0}}-1\right) t\left(l_{0}\right) \tau-t\left(k_{0}\right) t\left(l_{0}\right) \tau^{2}
\end{array}\right) \quad(\text { if } m=1),
\end{aligned}
$$


where $t(-k)=-t(k)$ implies the last equality. Let $P, Q$ and $R$ be non-zero rational numbers such that

$$
\vec{r}(w)=\left(\begin{array}{c}
\cdots+Q \tau^{2 m-1} \\
\ldots+P \tau^{2 m-1}+R \tau^{2 m}
\end{array}\right) .
$$

Then $R / P=2^{k_{0}}+1$ and $R / Q=2^{l_{m-1}}+1$. On the other hand, if

$$
\left(\begin{array}{c}
\ldots+Q \tau^{2 m-1} \\
\ldots+P \tau^{2 m-1}+R \tau^{2 m}
\end{array}\right), \quad\left(\begin{array}{c}
\ldots+Q^{\prime} \tau^{2 m^{\prime}-1} \\
\ldots+P^{\prime} \tau^{2 m^{\prime}-1}+R^{\prime} \tau^{2 m^{\prime}}
\end{array}\right)
$$

are linearly dependent over $\mathbb{Q}\left(\tau^{2}\right)$ then

$$
P: Q: R=P^{\prime}: Q^{\prime}: R^{\prime} \text {. }
$$

Hence, for

$$
w=u^{k_{0}} v^{l_{0}} \ldots v^{l_{m-1}} \quad \text { and } \quad w^{\prime}=u^{k_{0}^{\prime}} v^{l_{0}^{\prime}} \ldots v^{l_{m^{\prime}-1}^{\prime}},
$$

if $k_{0} \neq k_{0}^{\prime}$ or $l_{m-1} \neq l_{m^{\prime}-1}^{\prime}$ then $\vec{r}(w)$ and $\vec{r}\left(w^{\prime}\right)$ are linearly independent over $\mathbb{Q}\left(\tau^{2}\right)$.

By the remarks preceding Proposition 0 and Lemma 3 this concludes the proof of the Theorem.

Proof of the Corollary. It remains to show a proposition stated in the Introduction prior to the Corollary.

Proposition 1. The set of n-tuples $\left(\alpha_{0}, \ldots, \alpha_{n-1}\right)$ in $\left(S A_{2}(\mathbb{R})\right)^{n}$ which fail to be free generators of a free locally commutative subgroup is a subset of a countable union of proper algebraic subsets.

Proof. First we mention that $v, u^{-1} v u, u^{-2} v u^{2}, \ldots$ generate a free subgroup of infinite rank. Let $w$ be a non-empty reduced word in the letters $X_{0}^{-1}, \ldots, X_{n-1}^{-1}, X_{0}, \ldots, X_{n-1}$. For $\alpha_{0}, \ldots, \alpha_{n-1}$ in $S A_{2}(\mathbb{R})$, the transformation $w\left(\alpha_{0}, \ldots, \alpha_{n-1}\right)$ of $S A_{2}(\mathbb{R})$ is the value of the map $\left(S A_{2}(\mathbb{R})\right)^{n} \rightarrow$ $S A_{2}(\mathbb{R})$ defined by the word $w$ (e.g., $w\left(\alpha_{0}, \alpha_{1}, \alpha_{2}\right)=\alpha_{0}^{-1} \alpha_{1}^{-1} \alpha_{0}^{2} \alpha_{2}^{3}$ for $\left.w=X_{0}^{-1} X_{1}^{-1} X_{0}^{2} X_{2}^{3}\right)$. For each $w$, the set

(๒) $\quad\left\{\left(\alpha_{0}, \ldots, \alpha_{n-1}\right) \in\left(S A_{2}(\mathbb{R})\right)^{n}: w\left(\alpha_{0}, \ldots, \alpha_{n-1}\right)=\operatorname{id}\right.$ in $\left.S A_{2}(\mathbb{R})\right\}$

is a proper algebraic subset of $\left(S A_{2}(\mathbb{R})\right)^{n}$. In particular, by the Theorem, the $n$-tuple $\left(v, u^{-1} v u, \ldots, u^{-n+1} v u^{n-1}\right)$ does not belong to it.

Let $w, w^{\prime}$ be two words such that $w w^{\prime} \neq w^{\prime} w$. If $w\left(\alpha_{0}, \ldots, \alpha_{n-1}\right)$ and $w^{\prime}\left(\alpha_{0}, \ldots, \alpha_{n-1}\right)$ have a common fixed point $\vec{z}$, then

$$
\begin{aligned}
\vec{\xi}_{w\left(\alpha_{0}, \ldots, \alpha_{n-1}\right)} & =\left(\mathrm{id}-M_{w\left(\alpha_{0}, \ldots, \alpha_{n-1}\right)}\right) \vec{z}, \\
\vec{\xi}_{w^{\prime}\left(\alpha_{0}, \ldots, \alpha_{n-1}\right)} & =\left(\mathrm{id}-M_{w^{\prime}\left(\alpha_{0}, \ldots, \alpha_{n-1}\right)}\right) \vec{z},
\end{aligned}
$$

where $M_{\gamma}$ is the linear part and $\vec{\xi}_{\gamma}$ is the translation part of $\gamma$. So we have 


$$
\begin{aligned}
& \operatorname{det}\left(\mathrm{id}-M_{w^{\prime}\left(\alpha_{0}, \ldots, \alpha_{n-1}\right)}\right) \operatorname{adj}\left(\mathrm{id}-M_{w\left(\alpha_{0}, \ldots, \alpha_{n-1}\right)}\right) \vec{\xi}_{w\left(\alpha_{0}, \ldots, \alpha_{n-1}\right)} \\
& =\operatorname{det}\left(\mathrm{id}-M_{w^{\prime}\left(\alpha_{0}, \ldots, \alpha_{n-1}\right)}\right) \operatorname{adj}\left(\mathrm{id}-M_{w\left(\alpha_{0}, \ldots, \alpha_{n-1}\right)}\right)\left(\mathrm{id}-M_{w\left(\alpha_{0}, \ldots, \alpha_{n-1}\right)}\right) \vec{z} \\
& =\operatorname{det}\left(\mathrm{id}-M_{w^{\prime}\left(\alpha_{0}, \ldots, \alpha_{n-1}\right)}\right) \operatorname{det}\left(\mathrm{id}-M_{w\left(\alpha_{0}, \ldots, \alpha_{n-1}\right)}\right) \vec{z} \\
& =\operatorname{det}\left(\mathrm{id}-M_{w\left(\alpha_{0}, \ldots, \alpha_{n-1}\right)}\right) \operatorname{adj}\left(\mathrm{id}-M_{w^{\prime}\left(\alpha_{0}, \ldots, \alpha_{n-1}\right)}\right)\left(\mathrm{id}-M_{w^{\prime}\left(\alpha_{0}, \ldots, \alpha_{n-1}\right)}\right) \vec{z} \\
& =\operatorname{det}\left(\mathrm{id}-M_{w\left(\alpha_{0}, \ldots, \alpha_{n-1}\right)}\right) \operatorname{adj}\left(\mathrm{id}-M_{w^{\prime}\left(\alpha_{0}, \ldots, \alpha_{n-1}\right)}\right) \vec{\xi}_{w^{\prime}\left(\alpha_{0}, \ldots, \alpha_{n-1}\right)},
\end{aligned}
$$

where

$$
\operatorname{adj}\left(\begin{array}{ll}
a & b \\
c & d
\end{array}\right)=\left(\begin{array}{cc}
d & -b \\
-c & a
\end{array}\right)
$$

Hence the set

$$
\begin{array}{r}
(\sharp) \quad\left\{\left(\alpha_{0}, \ldots, \alpha_{n-1}\right) \in\left(S A_{2}(\mathbb{R})\right)^{n}: w\left(\alpha_{0}, \ldots, \alpha_{n-1}\right) \text { and } w^{\prime}\left(\alpha_{0}, \ldots, \alpha_{n-1}\right)\right. \\
\text { have a common fixed point in } \left.\mathbb{R}^{2}\right\}
\end{array}
$$

is contained in a proper algebraic subset (proper, since by the Theorem, $\left(v, u^{-1} v u, \ldots, u^{-n+1} v u^{n-1}\right)$ does not belong to it). So, the union of all sets represented by $(\emptyset)$ and $(\sharp)$,

$\left\{\left(\alpha_{0}, \ldots, \alpha_{n-1}\right) \in\left(S A_{2}(\mathbb{R})\right)^{n}: \alpha_{0}, \ldots, \alpha_{n-1}\right.$ do not generate a free group which acts on $\mathbb{R}^{2}$ in a locally commutative way\}, is a subset of a countable union of proper algebraic subsets of $\left(S A_{2}(\mathbb{R})\right)^{n}$.

\section{References}

[K] K. Kuratowski, Applications of the Baire-category method to the problem of independent sets, Fund. Math. 81 (1973), 65-72.

[L] M. Laczkovich, Paradoxical sets under $S L_{2}(\mathbb{R})$, Ann. Univ. Sci. Budapest Rolando Eötvös Sect. Math. 42 (1999), 141-145.

$\left[\mathrm{M}_{0}\right]$ J. Mycielski, About sets invariant with respect to denumerable changes, Fund. Math. 45 (1958), 296-305.

$\left[\mathrm{M}_{1}\right] \quad-$, Independent sets in topological algebras, ibid. 55 (1964), 139-147.

$\left[\mathrm{M}_{2}\right] \quad-$, Almost every function is independent, ibid. 81 (1973), 43-48.

$\left[\mathrm{M}_{3}\right] \quad-$, Non-amenable groups with amenable action and some paradoxical decompositions in the plane, Colloq. Math. 75 (1998), 149-157.

[MW] J. Mycielski and S. Wagon, Large free groups of isometries and their geometrical uses, Enseign. Math. 30 (1984), 247-267.

$\left[\mathrm{S}_{0}\right] \quad$ K. Satô, A free group acting on $\mathbb{Z}^{2}$ without fixed points, Enseign. Math. 45 (1999), 189-194.

$\left[\mathrm{S}_{1}\right] \quad-$, Free isometric actions on the affine space $\mathbb{Q}^{n}$, Indag. Math. 13 (2002), 389405.

[V] J. von Neumann, Zur allgemeinen Theorie des Maßes, Fund. Math. 13 (1929), 73-116. 
[W] S. Wagon, The Banach-Tarski Paradox, Cambridge Univ. Press, Cambridge, 1985.

Department of Mathematics

Faculty of Engineering

Tamagawa University

6-1-1, Tamagawa-Gakuen, Machida

Tokyo 194-8610, Japan

E-mail: kenzi@eng.tamagawa.ac.jp

Received 1 April 2003;

in revised form 24 September 2003 